DFTT-18/93

\title{
A NOTE ON QUANTUM STRUCTURE CONSTANTS
}

\author{
Leonardo Castellani * and Marco A. R-Monteiro ${ }^{\diamond *}$ \\ * Istituto Nazionale di Fisica Nucleare, Sezione di Torino \\ and \\ Dipartimento di Fisica Teorica \\ Via P. Giuria 1, 10125 Torino, Italy. \\ ${ }^{\diamond}$ Centro Brasileiro de Pesquisas Fisicas (CBPF) \\ Rio de Janeiro, Brasil
}

\begin{abstract}
The Cartan-Maurer equations for any $q$-group of the $A_{n-1}, B_{n}, C_{n}, D_{n}$ series are given in a convenient form, which allows their direct computation and clarifies their connection with the $q=1$ case. These equations, defining the field strengths, are essential in the construction of $q$-deformed gauge theories. An explicit expression $\omega^{i} \wedge \omega^{j}=-Z^{i j}{ }_{k l} \omega^{k} \wedge \omega^{l}$ for the $q$-commutations of left-invariant one-forms is found, with $Z_{k l}^{i j} \omega^{k} \wedge \omega^{l} \stackrel{q \rightarrow 1}{\longrightarrow} \omega^{j} \wedge \omega^{i}$.
\end{abstract}

DFTT-18/93

April 1993

email addresses: Decnet $=31890:$ :castellani;

Bitnet= castellani@to.infn.it 
Quantum groups [1]- [4] appear as a natural and consistent algebraic structure behind continuously deformed physical theories. Thus, in recent times, there have been various proposals for deformed gauge theories and gravity-like theories [5] based on $q$-groups.

Such deformations are interesting from different points of view, depending also on which theory we are deforming. For example, in quantized q-gravity theories space-time becomes noncommutative, a fact that does not contradict (Gedanken) experiments under the Planck length, and that could possibly provide a regularization mechanism [7, 8]. On the other hand, for the q-gauge theories constructed in [6] spacetime can be taken to be the ordinary Minkowski spacetime, the $q$ commutativity residing on the fiber itself. As shown in [6], one can construct a $q$-lagrangian invariant under $q$-gauge variations. This could suggest a way to break the classical symmetry via a $q$-deformation, rather than by introducing ad hoc scalar fields. Note also that, unlike the $q=1$ case, the $q$-group $U_{q}(N)$ is simple, thus providing a "quantum unification" of $S U(N) \otimes U(1)$.

In order to proceed from the algebraic $q$-structure to a dynamical $q$-field theory, it is essential to investigate the differential calculus on $q$-groups. Indeed this provides the $q$-analogues of the "classical" definitions of curvatures, field strengths, exterior products of forms, Bianchi identities, covariant and Lie derivatives and so on, see for ex. [9] for a review.

In this Letter we address and solve a specific problem: to find the Cartan-Maurer equations for any $q$-group of the $A, B, C, D$ series in explicit form. These equations define the field strengths of the corresponding $q$-gauge theories [6]. The $A_{n-1}$ case was already treated in [9], where the structure constants were given explicitly, and shown to have the correct classical limit.

To our knowledge, this problem has been tackled previously only in ref. [10]. There, however, the authors use (for the $B, C, D$-groups) a definition for the exterior product different from the one introduced in ref.s [11], adopted in [12, 13, 9] and in the present Letter. As we will comment later, their choice leads to a more complicated scenario.

Quantum groups are characterized by their $R$-matrix, which controls the noncommutativity of the quantum group basic elements $T^{a}{ }_{b}$ (fundamental representation):

$$
R^{a b}{ }_{e f} T^{e}{ }_{c} T^{f}{ }_{d}=T^{b}{ }_{f} T^{a}{ }_{e} R^{e f}{ }_{c d}
$$

and satisfies the quantum Yang-Baxter equation

$$
R_{a_{2} b_{2}}^{a_{1} b_{1}} R_{a_{3} c_{2}}^{a_{2} c_{1}} R_{b_{3} c_{3}}^{b_{2} c_{2}}=R_{b_{2} c_{2}}^{b_{1} c_{1}} R_{a_{2} c_{3}}^{a_{1} c_{2}} R_{a_{3} b_{3}}^{a_{2} b_{2}},
$$

a sufficient condition for the consistency of the "RTT" relations (1). Its elements depend continuously on a (in general complex) parameter $q$, or even on a set of parameters. For $q \rightarrow 1$ we have $R^{a b}{ }_{c d} \stackrel{q \rightarrow 1}{\longrightarrow} \delta_{c}^{a} \delta_{d}^{b}$, i.e. the matrix entries $T^{a}{ }_{b}$ commute and become the usual entries of the fundamental representation. The 
$q$-analogue of $\operatorname{det} T=1$, unitarity and orthogonality conditions can be imposed on the elements $T_{b}^{a}$, consistently with the $R T T$ relations (11), see [3].

The (uniparametric) $R$-matrices for the $q$-groups of the $A_{n-1}, B_{n}, C_{n}, D_{n}$ series can be found in ref. [3]. We recall the projector decomposition of the $\hat{R}$ matrix defined by $\hat{R}_{c d}^{a b} \equiv R_{c d}^{b a}$, whose $q \rightarrow 1$ limit is the permutation operator $\delta_{d}^{a} \delta_{c}^{b}$ :

$A_{n}$ series:

$$
\hat{R}=q P_{+}-q^{-1} P_{-}
$$

with

$$
\begin{aligned}
& P_{+}=\frac{1}{q+q^{-1}}\left(\hat{R}+q^{-1} I\right) \\
& P_{-}=\frac{1}{q+q^{-1}}(-\hat{R}+q I) \\
& I=P_{+}+P_{-}
\end{aligned}
$$

$B_{n}, C_{n}, D_{n}$ series:

$$
\hat{R}=q P_{+}-q^{-1} P_{-}+\varepsilon q^{\varepsilon-N} P_{0}
$$

with

$$
\begin{aligned}
& P_{+}=\frac{1}{q+q^{-1}}\left[\hat{R}+q^{-1} I-\left(q^{-1}+\varepsilon q^{\varepsilon-N}\right) P_{0}\right] \\
& P_{-}=\frac{1}{q+q^{-1}}\left[-\hat{R}+q I-\left(q-\varepsilon q^{\varepsilon-N}\right) P_{0}\right] \\
& P_{0}=\frac{1-q^{2}}{\left(1-\varepsilon q^{N+1-\varepsilon}\right)\left(1+\varepsilon q^{\varepsilon-N+1}\right)} K \\
& K^{a b}{ }_{c d}^{a b} C_{c d} \\
& I=P_{+}+P_{-}+P_{0}
\end{aligned}
$$

where $\varepsilon=1$ for $B_{n}, D_{n}, \varepsilon=-1$ for $C_{n}$, and $N$ is the dimension of the fundamental representation $T^{a}{ }_{b}\left(N=2 n+1\right.$ for $B_{n}$ and $N=2 n$ for $\left.C_{n}, D_{n}\right) ; C_{a b}$ is the $q$-metric, and $C^{a b}$ its inverse (cf. ref. [3]).

From (3) and (5) we read off the eigenvalues of the $\hat{R}$ matrix, and deduce the characteristic equations:

$$
\begin{aligned}
& \left.(\hat{R}-q I)\left(\hat{R}+q^{-1} I\right)=0 \text { for } A_{n-1} \quad \text { (Hecke condition }\right) \\
& (\hat{R}-q I)\left(\hat{R}+q^{-1} I\right)\left(\hat{R}-\varepsilon q^{\varepsilon-N} I\right)=0, \text { for } B_{n}, C_{n}, D_{n}
\end{aligned}
$$

The differential calculus on $q$-groups, initiated in ref.s [11], can be entirely formulated in terms of the $R$ matrix. The general constructive procedure can be found in ref. [12], or, in the notations we adopt here, in ref. [9].

As discussed in 111 and [12, we can start by introducing the (quantum) leftinvariant one-forms $\omega_{a}^{b}$, whose exterior product

$$
\omega_{a_{1}}^{a_{2}} \wedge \omega_{d_{1}}^{d_{2}} \equiv \omega_{a_{1}}^{a_{2}} \otimes \omega_{d_{1}}^{d_{2}}-\left.\Lambda_{a_{1} d_{1}}^{a_{2} d_{2}}\right|_{c_{2} b_{2}} ^{c_{1} b_{1}} \omega_{c_{1}}^{c_{2}} \otimes \omega_{b_{1}}^{b_{2}}
$$

is defined by the braiding matrix $\Lambda$ :

$$
\left.\Lambda_{a_{1} d_{1}}^{a_{2} d_{2}}\right|_{c_{2} b_{2}} ^{c_{1} b_{1}} \equiv d^{f_{2}} d_{c_{2}}^{-1} \hat{R}_{c_{2} g_{1}}^{b_{1} f_{2}}\left(\hat{R}^{-1}\right)^{c_{1} g_{1}}{ }_{a_{1} e_{1}}\left(\hat{R}^{-1}\right)_{d_{1} g_{2}}^{a_{2} e_{1}} \hat{R}_{b_{2} f_{2}}^{d_{2} g_{2}}
$$


For $q \rightarrow 1$ the braiding matrix $\Lambda$ becomes the usual permutation operator and one recovers the classical exterior product. Note that the "quantum cotangent space" $\Gamma$, i.e. the space spanned by the quantum one-forms $\omega_{a}^{b}$, has dimension $N^{2}$, in general bigger than its classical counterpart $\left(\operatorname{dim} \Gamma=N^{2}\right.$ only for the $U_{q}(N)$ groups). This is necessary in order to have a bicovariant bimodule structure for $\Gamma$ (cf. ref. (《10.). The same phenomenon occurs for the $q$-Lie generators defined below. For these, however, one finds restrictions (induced by the conditions imposed on the $T_{b}^{a}$ elements) that in general reduce the number of independent generators. Working with $N^{2}$ generators is more convenient, since the nice quadratic relations (16) of the $q$-Lie algebra become of higher order if one expresses them in terms of a reduced set of independent generators. For a discussion see [13].

The relations (7) and (8) satisfied by the $\hat{R}$ matrices of the $A$ and $B, C, D$ series respectively reflect themselves in the relations for the matrix $\Lambda$ :

$$
\left(\Lambda+q^{2} I\right)\left(\Lambda+q^{-2} I\right)(\Lambda-I)=0
$$

for the $A$ q-groups, and

$$
\begin{aligned}
\left(\Lambda+q^{2} I\right)\left(\Lambda+q^{-2} I\right)\left(\Lambda+\varepsilon q^{\varepsilon+1-N} I\right)\left(\Lambda+\varepsilon q^{N-\varepsilon-1} I\right) & \\
& \times\left(\Lambda-\varepsilon q^{N+1-\varepsilon} I\right)\left(\Lambda-\varepsilon q^{-N-1+\varepsilon} I\right)(\Lambda-I)=0
\end{aligned}
$$

for the $B, C, D q$-groups, with the same $\varepsilon$ as in (8). We give later an easy proof of these two relations.

Besides defining the exterior product of forms, the matrix $\Lambda$ contains all the the information about the quantum Lie algebra corresponding to the $q$-group.

The exterior differential of a quantum k-form $\theta$ is defined by means of the biinvariant (i.e. left- and right-invariant) element $\tau=\sum_{a} \omega_{a}^{a}$ as follows:

$$
d \theta \equiv \frac{1}{q-q^{-1}}\left[\tau \wedge \theta-(-1)^{k} \theta \wedge \tau\right],
$$

The normalization $\frac{1}{q-q^{-1}}$ is necessary in order to obtain the correct classical limit (see for ex. 91). This linear map satisfies $d^{2}=0$, the Leibniz rule and commutes with the left and right action of the $q$-group [12].

The exterior differentiation allows the definition of the "quantum Lie algebra generators" $\chi_{a_{2}}^{a_{1}}$, via the formula [1]

$$
d a=\frac{1}{q-q^{-1}}[\tau a-a \tau]=\left(\chi_{a_{2}}^{a_{1}} * a\right) \omega_{a_{1}}^{a_{2}} .
$$

where

$$
\chi * a \equiv(i d \otimes \chi) \Delta(a), \quad \forall a \in G_{q}, \quad \chi \in G_{q}^{\prime}
$$

and $\Delta$ is the usual coproduct on the quantum group $G_{q}$, defined by $\Delta\left(T^{a}{ }_{b}\right) \equiv$ $T^{a}{ }_{c} \otimes T^{c}{ }_{b}$. The $q$-generators $\chi$ are linear functionals on $G_{q}$. By taking the exterior 
derivative of (14), using $d^{2}=0$ and the bi-invariance of $\tau=\omega_{b}^{b}$, we arrive at the $q$-Lie algebra relations [12], [9]:

$$
\chi_{d_{2}}^{d_{1}} \chi_{c_{2}}^{c_{1}}-\left.\Lambda_{e_{1} f_{1}}^{e_{2} f_{2}}\right|_{d_{2} c_{2}} ^{d_{1} c_{1}} \chi_{e_{2}}^{e_{1}} \chi_{f_{2}}^{f_{1}}=\left.\mathbf{C}_{d_{2} c_{2}}^{d_{1} c_{1}}\right|_{a_{1}} ^{a_{2}} \chi_{a_{2}}^{a_{1}}
$$

where the structure constants are explicitly given by:

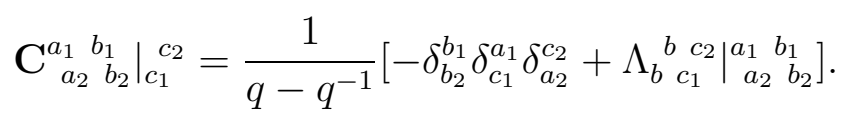

and $\chi_{d_{2}}^{d_{1}} \chi_{c_{2}}^{c_{1}} \equiv\left(\chi_{d_{2}}^{d_{1}} \otimes \chi_{c_{2}}^{c_{1}}\right) \Delta$. Notice that

$$
\left.\Lambda_{a_{1} d_{1}}^{a_{2} d_{2}}\right|_{c_{2} b_{2}} ^{c_{1} b_{1}}=\delta_{a_{1}}^{b_{1}} \delta_{b_{2}}^{a_{2}} \delta_{d_{1}}^{c_{1}} \delta_{c_{2}}^{d_{2}}+O\left(q-q^{-1}\right)
$$

because the $R$ matrix itself has the form $R=I+\left(q-q^{-1}\right) U$, with $U$ finite in the $q \rightarrow 1$ limit, see ref. [3]. Then it is easy to see that (17) has a finite $q \rightarrow 1$ limit, since the $\frac{1}{q-q^{-1}}$ terms cancel.

The Cartan-Maurer equations are found by applying to $\omega_{c_{1}}^{c_{2}}$ the exterior differential as defined in (13):

$$
d \omega_{c_{1}}^{c_{2}}=\frac{1}{q-q^{-1}}\left(\omega_{b}^{b} \wedge \omega_{c_{1}}^{c_{2}}+\omega_{c_{1}}^{c_{2}} \wedge \omega_{b}^{b}\right)
$$

Written as above, the Cartan-Maurer equations are not of much use for computations. The right-hand side has an undefined $\frac{0}{0}$ classical limit. We need a formula of the type $\omega_{c_{1}}^{c_{2}} \wedge \omega_{b}^{b}=-\omega_{b}^{b} \wedge \omega_{c_{1}}^{c_{2}}+O\left(q-q^{-1}\right)$ that allows to eliminate in (19) the terms with the trace $\omega_{b}^{b}$ (which has no classical counterpart) and obtain an explicitly $q \rightarrow 1$ finite expression.

The desired " $\omega$-permutator" can be found as follows. We first treat the case of the $A_{n-1}$ series. We apply relation (7) to the tensor product $\omega \otimes \omega$, i.e.:

$$
\left(\Lambda_{k l}^{i j}+q^{2} \delta_{k}^{i} \delta_{l}^{j}\right)\left(\Lambda_{m n}^{k l}+q^{-2} \delta_{m}^{k} \delta_{n}^{l}\right)\left(\Lambda_{r s}^{m n}-\delta_{r}^{m} \delta_{s}^{n}\right) \omega^{m} \otimes \omega^{n}=0
$$

where we have used the adjoint indices ${ }^{i} \leftrightarrow{ }_{a}^{b}, i \leftrightarrow{ }_{b}{ }_{b}$. Inserting the definition of the exterior product $\omega^{n} \wedge \omega^{n}=\omega^{m} \otimes \omega^{n}-\Lambda_{r s}^{m n} \omega^{r} \otimes \omega^{s}$ yields

$$
\left(\Lambda_{k l}^{i j}+q^{2} \delta_{k}^{i} \delta_{l}^{j}\right)\left(\Lambda_{m n}^{k l}+q^{-2} \delta_{m}^{k} \delta_{n}^{l}\right) \omega^{m} \wedge \omega^{n}=0
$$

Multiplying by $\Lambda^{-1}$ gives $\left(\Lambda+\left(q^{2}+q^{-2}\right) I+\Lambda^{-1}\right) \omega \wedge \omega$, or equivalently

$$
\begin{gathered}
\omega^{i} \wedge \omega^{j}=-Z_{k l}^{i j} \omega^{k} \wedge \omega^{l} \\
Z_{k l}^{i j} \equiv \frac{1}{q^{2}+q^{-2}}\left[\Lambda_{k l}^{i j}+\left(\Lambda^{-1}\right)_{k l}^{i j}{ }_{k l}\right] .
\end{gathered}
$$

cf. ref. [9]. The $\omega$-permutator $Z_{k l}^{i j}$ has the expected $q \rightarrow 1$ limit, that is $\delta_{l}^{i} \delta_{k}^{j}$. 
There is another way to deduce the permutator $Z$, based on projector methods, that we will use for the $B, C, D$ series. We first illustrate it in the easier $A$-case. Define

$$
\left.\left(P_{I}, P_{J}\right)_{a_{1} d_{1}}^{a_{2} d_{2}}\right|_{c_{2} b_{2}} ^{c_{1} b_{1}} \equiv d^{f_{2}} d_{c_{2}}^{-1} \hat{R}_{c_{2} g_{1}}^{b_{1} f_{2}}\left(P_{I}\right)_{a_{1} e_{1}}^{c_{1} g_{1}}\left(\hat{R}^{-1}\right)_{d_{1} g_{2}}^{a_{2} e_{1}}\left(P_{J}\right)_{b_{2} f_{2}}^{d_{2} g_{2}}
$$

with $\mathrm{I}, \mathrm{J}=+,-$, the projectors $P_{+}, P_{-}$being given in (田). The $\left(P_{I}, P_{J}\right)$ are themselves projectors, i.e.:

$$
\left(P_{I}, P_{J}\right)\left(P_{K}, P_{L}\right)=\delta_{I K} \delta_{J L}\left(P_{I}, P_{J}\right)
$$

Moreover

$$
(I, I)=I
$$

so that

$$
(I, I)=\left(P_{+}+P_{-}, P_{+}+P_{-}\right)=\left(P_{+}, P_{+}\right)+\left(P_{-}, P_{-}\right)+\left(P_{+}, P_{-}\right)+\left(P_{-}, P_{+}\right)=I
$$

Eq.s (25) and (26) are easy to prove by using (24) and the relation, valid for all $A, B, C, D$ q-groups:

$$
d^{f} d_{c}^{-1} \hat{R}^{b f}{ }_{c g}\left(\hat{R}^{-1}\right)^{c e}{ }_{b a}=\delta_{a}^{f} \delta_{g}^{e}
$$

Projectors similar to (24) were already introduced in ref. [10]. From the definition (10) of $\Lambda$, using (3) and (24) we can write

$$
\Lambda=\left(P_{+}, P_{+}\right)+\left(P_{-}, P_{-}\right)-q^{-2}\left(P_{+}, P_{-}\right)-q^{2}\left(P_{-}, P_{+}\right)
$$

This decomposition shows that $\Lambda$ has eigenvalues $1, q^{ \pm 2}$, and proves therefore eq. (11). From the definition of the exterior product $\omega \wedge \omega=\omega \otimes \omega-\Lambda \omega \otimes \omega$ we find the action of the projectors $\left(P_{I}, P_{J}\right)$ on $\omega \wedge \omega$ :

$$
\begin{gathered}
\left(P_{+}, P_{+}\right) \omega \wedge \omega=\left(P_{-}, P_{-}\right) \omega \wedge \omega=0 \\
\left(P_{+}, P_{-}\right) \omega \wedge \omega=\left(1+q^{-2}\right)\left(P_{+}, P_{-}\right) \omega \otimes \omega, \quad\left(P_{-}, P_{+}\right) \omega \wedge \omega=\left(1+q^{2}\right)\left(P_{-}, P_{+}\right) \omega \otimes \omega
\end{gathered}
$$

Using (27) and (30) we find :

$\omega \wedge \omega=\left[\left(P_{+}, P_{+}\right)+\left(P_{-}, P_{-}\right)+\left(P_{+}, P_{-}\right)+\left(P_{-}, P_{+}\right)\right] \omega \wedge \omega=\left[\left(P_{+}, P_{-}\right)+\left(P_{-}, P_{+}\right)\right] \omega \wedge \omega$

The $\omega$-permutator is therefore $Z=-\left(P_{+}, P_{-}\right)-\left(P_{-}, P_{+}\right)$. We can express it in terms of the $\Lambda$ matrix by observing that

$$
\left(P_{+}, P_{-}\right)+\left(P_{-}, P_{+}\right)=-\frac{1}{q^{2}+q^{-2}}\left(\Lambda+\Lambda^{-1}\right)+\frac{2}{q^{2}+q^{-2}}\left(\left(P_{+}, P_{+}\right)+\left(P_{-}, P_{-}\right)\right)
$$

as one deduces from (29). Note that $\Lambda^{-1}$ is given in terms of projectors by the same expression as in (29), with $q \rightarrow q^{-1}$. When acting on $\omega \wedge \omega$ the $\left(P_{+}, P_{+}\right),\left(P_{-}, P_{-}\right)$ terms in (33) can be dropped because of (30), so that finally we arrive at eq. (23). 
Because of the expansion (18) and a similar one for $\Lambda^{-1}$ we easily see that the $\omega$-permutator (23) can be expanded as

$$
\left.Z_{c_{1} d_{1}}^{c_{2} d_{2}}\right|_{a_{2} b_{2}} ^{a_{1} b_{1}} \omega_{a_{1}}^{a_{2}} \wedge \omega_{b_{1}}^{b_{2}}=\omega_{d_{1}}^{d_{2}} \wedge \omega_{c_{1}}^{c_{2}}+\left.\left(q-q^{-1}\right) W_{c_{1} d_{1}}^{c_{2} d_{2}}\right|_{a_{2} b_{2}} ^{a_{1} b_{1}} \omega_{a_{1}}^{a_{2}} \wedge \omega_{b_{1}}^{b_{2}}
$$

where $W$ is a finite matrix in the limit $q \rightarrow 1$.

Let us return to the Cartan-Maurer eqs. (19). Using (22) we can write:

$$
d \omega_{c_{1}}^{c_{2}}=\frac{1}{q-q^{-1}}\left(\omega_{b}^{b} \wedge \omega_{c_{1}}^{c_{2}}-\left.Z_{c_{1} b}^{c_{2} b}\right|_{a_{2} b_{2}} ^{a_{1} b_{1}} \omega_{a_{1}}^{a_{2}} \wedge \omega_{b_{1}}^{b_{2}}\right)
$$

where $Z$ is given by $\left(\Lambda+\Lambda^{-1}\right) /\left(q^{2}+q^{-2}\right)$, cf. (23). Because of (34) we see that the $\omega_{b}^{b}$ terms disappear, and (35) has a finite $q \rightarrow 1$ limit.

We now repeat the above construction for the case of $q$-groups belonging to the $B, C, D$ series.

Using (可) and (24) we find the following projector decomposition for the $\Lambda$ matrix :

$$
\begin{aligned}
\Lambda= & \left(P_{+}, P_{+}\right)+\left(P_{-}, P_{-}\right)+\left(P_{0}, P_{0}\right)+\varepsilon q^{\varepsilon-1-N}\left(P_{+}, P_{0}\right)+\varepsilon q^{-(\varepsilon-1-N)}\left(P_{0}, P_{+}\right) \\
& -q^{-2}\left(P_{+}, P_{-}\right)-q^{2}\left(P_{-}, P_{+}\right)-\varepsilon q^{N-\varepsilon-1}\left(P_{0}, P_{-}\right)-\varepsilon q^{-(N-\varepsilon-1)}\left(P_{-}, P_{0}\right)
\end{aligned}
$$

from which we read off the eigenvalues of $\Lambda$, and prove eq. (12). Proceeding as in the $A$ case, we find the action of the projectors on $\omega \wedge \omega$ :

$$
\begin{gathered}
\left(P_{+}, P_{+}\right) \omega \wedge \omega=\left(P_{-}, P_{-}\right) \omega \wedge \omega=\left(P_{0}, P_{0}\right) \omega \wedge \omega=0 \\
\left(P_{+}, P_{-}\right) \omega \wedge \omega=\left(1+q^{-2}\right)\left(P_{+}, P_{-}\right) \omega \otimes \omega, \\
\left(P_{-}, P_{+}\right) \omega \wedge \omega=\left(1+q^{2}\right)\left(P_{-}, P_{+}\right) \omega \otimes \omega \\
\left(P_{-}, P_{0}\right) \omega \wedge \omega=\left(1+\varepsilon q^{-(N-\varepsilon-1)}\right)\left(P_{-}, P_{0}\right) \omega \otimes \omega, \\
\left(P_{0}, P_{-}\right) \omega \wedge \omega=\left(1+\varepsilon q^{N-\varepsilon-1}\right)\left(P_{0}, P_{-}\right) \omega \otimes \omega \\
\left(P_{+}, P_{0}\right) \omega \wedge \omega=\left(1-\varepsilon q^{\varepsilon-1-N}\right)\left(P_{+}, P_{0}\right) \omega \otimes \omega, \\
\left(P_{0}, P_{+}\right) \omega \wedge \omega=\left(1-\varepsilon q^{-(\varepsilon-1-N)}\right)\left(P_{0}, P_{+}\right) \omega \otimes \omega
\end{gathered}
$$

Again the sum of the projectors $\left(P_{I}, P_{J}\right)$ yields the identity, so that we can write:

$$
\omega \wedge \omega=\left[\left(P_{+}, P_{-}\right)+\left(P_{-}, P_{+}\right)+\left(P_{-}, P_{0}\right)+\left(P_{0}, P_{-}\right)+\left(P_{+}, P_{0}\right)+\left(P_{0}, P_{+}\right)\right] \omega \wedge \omega
$$

where we have taken (37) into account. The $\omega$-permutator $Z$ is therefore given by

$$
Z=-\left[\left(P_{+}, P_{-}\right)+\left(P_{-}, P_{+}\right)+\left(P_{-}, P_{0}\right)+\left(P_{0}, P_{-}\right)+\left(P_{+}, P_{0}\right)+\left(P_{0}, P_{+}\right)\right]
$$


Can we express it in terms of odd powers of the $\Lambda$ matrix, as in the case of the $A$ groups ? The answer is: only partially. In fact, by elementary algebra we find that

$$
Z=-\alpha\left(\Lambda+\Lambda^{-1}\right)-\beta\left(\Lambda^{3}+\Lambda^{-3}\right)-\left(1-\alpha q_{-\varepsilon N}-\beta q_{-3 \varepsilon N}\right)\left[\left(P_{\sigma}, P_{0}\right)+\left(P_{0}, P_{\sigma}\right)\right]
$$

with $\sigma \equiv \operatorname{sgn}(\varepsilon)$ and

$$
\begin{gathered}
\alpha=-\frac{1+\beta q_{6}}{q_{2}} \\
\beta=\frac{q_{2}-q_{\varepsilon N-2}}{q_{6} q_{\varepsilon N-2}-q_{2} q_{3(\varepsilon N-2)}} \\
q_{n} \equiv q^{n}+q^{-n}
\end{gathered}
$$

Note: $\Lambda^{r}$ is given by

$$
\begin{aligned}
\Lambda^{r}=\left(P_{+}, P_{+}\right)+ & \left(P_{-}, P_{-}\right)+\left(P_{0}, P_{0}\right)+\varepsilon^{r}\left[q^{r(\varepsilon-1-N)}\left(P_{+}, P_{0}\right)+q^{-r(\varepsilon-1-N)}\left(P_{0}, P_{+}\right)\right] \\
& +(-1)^{r}\left[q^{-2 r}\left(P_{+}, P_{-}\right)+q^{2 r}\left(P_{-}, P_{+}\right)\right] \\
& +(-\varepsilon)^{r}\left[q^{r(N-\varepsilon-1)}\left(P_{0}, P_{-}\right)+q^{-r(N-\varepsilon-1)}\left(P_{-}, P_{0}\right)\right]
\end{aligned}
$$

Let us check that $Z$ in (43) has a correct classical limit. We have $\alpha \stackrel{q \rightarrow 1}{\longrightarrow}-\frac{9}{16}$ and $\beta \stackrel{q \rightarrow 1}{\longrightarrow} \frac{1}{16}$; taking into account that the $\left(P_{\sigma}, P_{0}\right),\left(P_{0}, P_{\sigma}\right)$ terms disappear in the classical limit (cf. eq. (39), (40)) when applied to $\omega \wedge \omega$, we find the expected limit $Z_{k l}^{i j} \stackrel{q \rightarrow 1}{\longrightarrow} \delta_{l}^{i} \delta_{k}^{j}$.

The Cartan-Maurer equations are deduced as before, and are given by (35) where now $Z$ is the $\omega$ permutator of eq. (43) (Note: for explicit calculations the expression (42) or equivalently $Z=\left(P_{+}, P_{+}\right)+\left(P_{-}, P_{-}\right)+\left(P_{0}, P_{0}\right)-I$ is more convenient). Again the $\omega_{b}^{b}$ terms drop out since $Z$ admits the expansion $\left.Z_{c_{1} d_{1}}^{c_{2} d_{2}}\right|_{a_{2} b_{2}} ^{a_{1} b_{1}} \omega_{a_{1}}^{a_{2}} \wedge \omega_{b_{1}}^{b_{2}}=$ $-\omega_{d_{1}}^{d_{2}} \wedge \omega_{c_{1}}^{c_{2}}+O\left(q-q^{-1}\right)$.

In conclusion: we have found an explicit (and computable) expression for the Cartan-Maurer equations of the $B_{n}, C_{n}, D_{n} q$-groups. This opens the possibility of constructing gauge theories of these $q$-groups, following the procedure used in [6] for the $A_{n-1} q$-groups.

Finally, let us comment on the differential calculus presented by the authors of ref. [10]. Their definition of exterior product in the $B, C, D$ case differs from ours (and from the one adopted in [11, 12, 13]), and essentially amounts to require that $\left(P_{\sigma}, P_{0}\right) \omega \wedge \omega=0,\left(P_{0}, P_{\sigma}\right) \omega \wedge \omega=0$, besides (37). This has one advantage: the term $\left(P_{\sigma}, P_{0}\right)+\left(P_{0}, P_{\sigma}\right)$ disappears in the expression (43). The disadvantage is that the defining formula $\omega \wedge \omega=(I-\Lambda) \omega \otimes \omega$ does not hold any more for the $B, C, D$ series, so that the general treatment of ref. [11] and the constructive procedure of ref.s [12] do not apply. 


\section{References}

[1] V. Drinfeld, Sov. Math. Dokl. 32 (1985) 254.

[2] M. Jimbo, Lett. Math. Phys. 10 (1985) 63; 11 (1986) 247.

[3] L.D. Faddeev, N.Yu. Reshetikhin and L.A. Takhtajan, Algebra and Analysis, 1 (1987) 178.

[4] S. Majid, Int. J. Mod. Phys. A5 (1990) 1.

[5] A.P. Isaev and Z. Popowicz, q-Trace for the Quantum Groups and q-deformed Yang-Mills Theory, Wroclaw preprint ITP UWr 786-91; I.Y. Aref'eva and I.V. Volovich, Mod. Phys. Lett A6 (1991) 893; Phys. Lett. B264 (1991) 62; L. Castellani, Phys. Lett. B279 (1992) 291 and Phys. Lett. B292 (1992) 93; S. Meljanac and S. Pallua, Classical field lagrangian and deformed algebras, DFPF/92/TH/15; A. Dimakis and F. Müller-Hoissen, Noncommutative differential calculus, gauge theory and gravitation, GOET-TP-33/92; K. Wu and R.-J. Zhang, Commun. Theor. Phys. 17 (1992) 175; T. Brzeziński and S. Majid, Quantum group gauge theory on quantum spaces, DAMTP/92-27 and Quantum group gauge theory on classical spaces, DAMTP/92-51.

[6] L. Castellani, $U_{q}(N)$ gauge theories, Torino preprint DFTT 74/92.

[7] A. Connes, Publ. Math. IHES Vol. 62 (1986) 41; Géométrie non commutative (Inter Editions, Paris, 1990).

[8] S. Majid, Class. Quantum Grav. 5 (1988) 1587; Int. J. Mod. Phys. A5 (1990) 4689 .

[9] P. Aschieri and L. Castellani, An introduction to non-commutative differential geometry on quantum groups, preprint CERN-TH.6565/92, DFTT-22/92 (1992), to be publ. in Int. J. Mod. Phys. A.

[10] U. Carow-Watamura, M. Schlieker, S. Watamura and W. Weich, Commun. Math. Phys. 142 (1991) 605.

[11] S.L. Woronowicz, Publ. RIMS, Kyoto Univ., Vol. 23, 1 (1987) 117; Commun. Math. Phys. 111 (1987) 613 and Commun. Math. Phys. 122, (1989) 125.

[12] B. Jurčo, Lett. Math. Phys. 22 (1991) 177.

[13] B. Zumino, Introduction to the Differential Geometry of Quantum Groups, LBL-31432 and UCB-PTH-62/91, notes of a plenary talk given at the 10-th IAMP Conf., Leipzig (1991); P. Schupp, P. Watts and B. Zumino, Bicovariant quantum algebras and quantum Lie algebras, LBL-32315, UCB-PTH-92/14. 